8 AN EVALUATION OF END OF LIFE CARE FOR PATIENTS WITH COVID-19 RECEIVING NON-INVASIVE PULMONARY SUPPORT

Elizabeth Woods, Kalyani Snell, Elizabeth Zabrocki, Elizabeth Reed. Newcastle Hospitals NHS Foundation Trust

\subsection{6/spcare-2021-PCC.26}

Background Non-invasive pulmonary support (NIPS), (CPAP or NIV), is available to patients with Covid-19 and a ward based ceiling of treatment. Evidence demonstrate a 50\% survival with NIPS in this cohort. We, and our respiratory colleagues, were interested to understand the experience of dying in this context.

Method This was a retrospective case note review. Aims and objectives include:

- To describe the symptoms experienced, medications required and reasons for withdrawal in patients dying of covid-19 following treatment with NIPS

- To evaluate care against the five priorities (NICE guideline (NG142)).

Results 18 patients were included for analysis. The majority were aged over 80 (67\%). All patients experienced breathlessness when dying, and seventeen had agitation or delirium. Twelve patients (66\%) required a regular benzodiazepine, either alone $(22 \%)$ or in combination with an opioid (45\%). Two patients (11\%) were treated with only an opioid. The doses of opioids and midazolam were relatively small - most commonly $10 \mathrm{mg}$. $66 \%$ of patients received $<3$ as required doses of opioid or midazolam in the final 24 hours. The commonest reasons for withdrawal were the patient stopping tolerating treatment $(56 \%)$, and treatment failure (28\%). No patients died within three hours of withdrawal, with the majority dying six hours to two days later. In 17 cases (94\%) it was recognised and documented that the patient was sick enough to die. This was communicated to the patient and/or their family in all 94\%. All patients had a DNACPR and Treatment Escalation Plan. 94\% of families were offered to visit their dying relative, this was taken up in $44 \%$ of cases.

Conclusions Good end of life care is achievable in the context of patients with Covid-19, receiving NIPs. Key learning includes:

- The need to regularly review symptoms and consider increasing background sc infusions more frequently than our usual practice of every 24 hrs.

\section{DELIVERING GOOD QUALITY END OF LIFE CARE (EOLC) DURING A PANDEMIC: THE RESPONSE OF THE TRANSFORMING END OF LIFE CARE TEAM (TEOLCT) TO COVID-19 IN AN ACUTE LONDON NHS TRUST}

\footnotetext{
${ }^{1}$ Gehan Soosaipillai, ${ }^{2}$ Joanne Bennetts, 'Madeleine McMillan, ${ }^{1}$ Lina Pereira, ${ }^{1}$ Finn Padmore, ${ }^{1}$ Sadaf Iqbal, ${ }^{2}$ Emily Collis. ${ }^{1}$ University College London Hospitals NHS Foundation Trust
} (UCLH): ${ }^{2}$ Central and North West London NHS Foundation Trust (CNWL)

10.1136/spcare-2021-PCC.27

Introduction The COVID-19 pandemic highlighted the need for high quality EOLC, unprecedented in scale and setting. We describe the initiatives led by the UCLH TEOLCT who played a key role in preparing and supporting staff to provide EOLC, as well as providing support for inpatients and their families.

Methods Utilising QI methodology, the TEOLCT rapidly implemented changes in six key areas of practice between 23/ $03 / 2020$ and 25/08/2020. The multidisciplinary TEOLCT collaborated with Specialist Palliative Care and Clinical Psychology teams to achieve these outcomes.

Results (i) Staff education: high demand for teaching, e.g. difficult conversations, EOLC and COVID-19 specific symptom control, for redeployed staff largely inexperienced in EOLC. 1037 clinical staff were trained utilising a combination of socially distanced lectures and video-conferencing/webinars. (ii) Staff support: drop-in sessions were facilitated for $>200$ staff members. (iii) Guidance and Standard Operating Procedures: for symptom control, non-invasive ventilation withdrawal and communicating with family were collaboratively written and disseminated with appropriate training. (iv) Clinical audit: quality of decision-making and documentation scrutinised by auditing treatment escalation plans and do not attempt cardiopulmonary resuscitation orders, identifying areas of practice improvement and training needs. (v) Clinical support: modifying the SWAN model of care for patients in last days of life, TEOLCT supported care of 107 patients during the pandemic peak (23/03 “ 15/05/2020), totalling 255 inpatient visits. (vi) Bereavement support: with restricted visiting and changes to after death care, TEOLCT oversaw formal bereavement support for bereaved families of 348/392 patients who died, plus appropriate sign-posting to community services.

Conclusions The TEOLCT rapidly adapted to an unprecedented clinical challenge, identifying and responding to needs, working towards a common goal and leading a coordinated response to the demand for training and support. The key areas of development will inform future practice to ensure ongoing training and support in future surges.

\section{WHAT CAN WE LEARN FROM THE COVID-19 PANDEMIC ABOUT ADMISSIONS TO A HOSPICE IPU?}

Emily Meeks, Liz Taylor. Hospice of the Good Shepherd

\subsection{6/spcare-2021-PCC.28}

Background The 2020 COVID-19 pandemic has affected how hospices have functioned, with visiting restrictions and perceived public fear of healthcare environments. It was thought that hospice IPU admissions had reduced and that those admitted were further on in their disease trajectory.

Aims To review hospice referrals, admissions, length of stay, care and communication records and mortality during the COVID-19 pandemic and compare it to the previous year.

Methods A retrospective review of the case notes of all admissions to a hospice IPU over a 6-month period from 1st of April to 30th September in 2019 and 2020.

Results There were 97 admissions in 2019 vs 94 in 2020. $44 / 97$ (45\%) vs 45/94 (48\%) died during admission. The average length of stay was 14.4 days (2019) vs 10.4 (2020) $\mathrm{p}=0.036$, when excluding respite admissions (which had temporarily suspended in 2020) and day procedure admissions the average length of stay was 17.3 days vs 10.3 days, $p=$ 
0.0009. Those referred in for end of life care was 27 vs 35 . Of those that died during their admission there was no difference in the length of stay 12.2 vs 10.1 days, $\mathrm{p}=0.41$. There was no significant difference in length of stay prior to starting the care and communication record, 9.4 vs 5.6 days, $\mathrm{p}=0.06$.

Conclusions Though there were no differences in numbers of admission during the COVID 19 pandemic. There were significant differences in the length of stay. It was thought that the those coming in during the pandemic were more likely to be coming in for end of life care but as no significant difference between length of stay of patients that died nor in how quickly they were started on the care and communication record this was not the case. This information should be considered when improving current discharge processes.

\section{EXPERIENCE OF COVID-19 IN A HOSPICE INPATIENT UNIT: CHARACTERISTICS, SYMPTOMS AND ETHICAL ISSUES}

Emma Gardner, Emma Tempest. St Helena Hospice

\subsection{6/spcare-2021-PCC.29}

Objectives One of the established risk factors for death from coronavirus disease 2019 (COVID-19) is comorbidity. Patients referred to palliative care before developing COVID-19 infection are therefore more vulnerable due to their pre-existing conditions. This study aimed to describe the impact of COVID-19 infection on hospice inpatients.

Methods A retrospective review was performed of patients with confirmed COVID-19 during an admission to one hospice inpatient unit in the five months between 1st March 2020 and 31st July 2020.

Results 12 patients were identified with confirmed COVID19. Mortality during the admission was $67 \% \quad(n=8)$. Patients had a median age of 74 , were $75 \%$ male and had a median Australia-modified Karnofsky Performance Status (AKPS) of 30. 100\% had comorbidities, most commonly malignancy (58\%), hypertension $(50 \%)$ and diabetes $(50 \%)$. The symptoms most frequently recorded were confusion (67\%), agitation (67\%), fever (50\%), cough (50\%) and breathlessness (50\%). Symptoms at the end of life were treated with subcutaneous infusion (100\%) using commonly prescribed medications (oxycodone used in 75\%, morphine $13 \%$, midazolam 88\%, levomepromazine 63\%, glycopyrronium 88\%, haloperidol 13\%) and doses. A third of cases were detected by a routine COVID-19 swab, however this policy led to ethical debate around testing in the last days of life.

Conclusions This small case series suggests that larger studies into symptoms of COVID-19 at the end of life and their control would be helpful, particularly if they take into account baseline (and the impact on those with palliative conditions or frailty) and collect data from community settings as well as hospital care. We understand that there is heterogeneity in COVID-19 testing policies at hospices across the UK, and feel further discussion around the ethical questions raised by testing, particularly at the end of life, would be beneficial in finding our way forward in this ever-changing pandemic landscape.

\section{REFLECTIONS ON PALLIATIVE CARE HEALTH CARE PROFESSIONALS' NEEDS DURING THE FIRST WAVE OF THE COVID-19 PANDEMIC 'THEMATIC ANALYSIS OF A POSTGRADUATE COHORT OF STUDENTS' DISCUSSION BOARD}

Fiona Rawlinson, Ross Smith, Toby Dinnen, Ed Hayes, Karianne Harper, Tony Duffy, Tamarha Jones, Lucy Thomas, Jo Clarke, Anjum Joad, Rajam lyer, Rachael Marcheant, Melissa Day, Matt Hooper, Lisa Bailey, Gayathri Subramaniam, Deepa Khurana, Wendy Wolfe, Sivakumar lyer, Lipika Patra, Daniel Barry, Diana Lees, Heidi Leeder, Catriona McKeating, Sian Gallard. Cardiff University

\subsection{6/spcare-2021-PCC.30}

background/Introduction The Covid-19 pandemic created immense unexpected pressure on postgraduate health care professional students undertaking academic study. The role of a community of practice within an international cohort of postgraduate students became apparent as the pandemic progressed. Needing a supported space away from social media in which to discuss developing issues, and a forum in which useful resources could be shared swiftly became apparent. A closed 'Discussion board' facility was used within the virtual learning platform in which themes and collections of support, ideas and materials were developed.

Methods Qualitative thematic analysis of anonymised contributions to the Discussion board 25th March 2020 - 31st July 2020. Permission was sought retrospectively from all participants on the Discussion Board; any information from those not willing for their content to be included was deleted from the transcripts before review. The transcripts were reviewed by 2 independent researchers for content and context.

Results 13 themes developed. Sharing resources (March); ethical issues, communication skills (including virtual communication), symptom control, team support and leadership, the challenge of covid 19 in remote communities, the collateral damage of the pandemic for children, involving the family remotely and maintaining one's own wellbeing in a pandemic ( April); Developing services, moving forward and quality improvement ( May); moral courage and managing the symptom of breathlessness (June).

Conclusions The generation of themes reflected the unprecedented challenges of the phase of the pandemic being experienced. Symptom control, communication skills and ethics leading to their application in different settings and the need after the first few weeks for team support, mental resilience and leadership. Valuable reflections and resources have been developed to help support future surges.

\section{SUPPORTING COMMUNITY PALLIATIVE CARE RESOURCES IS VITAL IN THE CONTINUING PANDEMIC}

Fiona Rawlinson, Margred Capel, James Davies, Susan Rees. City Hospice, Cardiff

\subsection{6/spcare-2021-PCC.31}

Background Covid-19 had a dramatic effect on the delivery of health care across all settings: institutions reduced footfall and strict social distancing measures were introduced. Face to face consultations were reduced; referrals to a community palliative care team (CPCT) increased. Referral data was explored for trends in referral patterns that might inform optimal use of resources. 